\title{
Work-in-Progress: Verifiably Safe SCUBA Diving using Commodity Sensors
}

\author{
Viren Bajaj \\ Carnegie Mellon University \\ Pittsburgh, Pennsylvania \\ Nathan Fulton ${ }^{*}$ \\ Carnegie Mellon University \\ Pittsburgh, Pennsylvania
}

\author{
Karim Elmaaroufi \\ Carnegie Mellon University \\ Pittsburgh, Pennsylvania \\ André Platzer \\ Carnegie Mellon University \\ Pittsburgh, Pennsylvania
}

\begin{abstract}
SCUBA diving is an activity in which divers remain underwater for prolonged periods by using a self-contained breathing apparatus. Diving is safety critical because changing depth too rapidly or running out of oxygen before surfacing can result in life-threatening consequences. These risks are currently minimized by using a wrist-mounted, 'air-integrated' dive computer that monitors time, depth and air tank pressure (received through expensive wireless transceivers or a hose). These computers are costly for the average recreational dive.

We present a hybrid systems model and safety proof for a SCUBA diving computer that estimates air consumption of the diver using commodity heart rate sensors, instead. We employ a mathematical model of oxygen uptake in response to exercise and thereby predict the time remaining for the air supply to be depleted, as well as obviate the need for a tank-mounted wireless transmitter or the cumbersome hose integrated computer for directly monitoring tank pressure. We formally verify a controller that ensures the diver can always surface without running out of breathable air.
\end{abstract}

\section{ACM Reference Format:}

Viren Bajaj, Karim Elmaaroufi, Nathan Fulton, and André Platzer. 2019. Work-in-Progress: Verifiably Safe SCUBA Diving using Commodity Sensors. In 2019 International Conference on Embedded Software Companion (EMSOFT'19 Companion), October 13-18, 2019, New York, NY, USA. ACM, New York, NY, USA, 2 pages. https://doi.org/10.1145/3349568.3351554

\section{INTRODUCTION}

When planning a SCUBA dive, divers must balance two conflicting safety constraints. They must arrive at the surface before exhausting their supply of oxygen, but must not ascend so quickly that it causes life-threatening dysbarism. Wearable SCUBA dive computers can help SCUBA divers balance these two safety constraints by alerting

\footnotetext{
${ }^{*}$ Also with MIT-IBM Watson AI Lab, IBM Research.

Permission to make digital or hard copies of all or part of this work for personal or classroom use is granted without fee provided that copies are not made or distributed for profit or commercial advantage and that copies bear this notice and the full citation on the first page. Copyrights for components of this work owned by others than the author(s) must be honored. Abstracting with credit is permitted. To copy otherwise, or republish, to post on servers or to redistribute to lists, requires prior specific permission and/or a fee. Request permissions from permissions@acm.org.

EMSOFT'19 Companion, October 13-18, 2019, New York, NY, USA

(C) 2019 Copyright held by the owner/author(s). Publication rights licensed to ACM. ACM ISBN 978-1-4503-6924-4/19/10 ..\$15.00

https://doi.org/10.1145/3349568.3351554
}

the diver when an ascent must begin to ensure that the diver can ascend at a safe rate without running out of breathable air.

The most common form of SCUBA dive computers achieves this by estimating the diver's nitrogen consumption using time and depth. Separately, the diver has to monitor their remaining air pressure via a gauge. It is up to the diver to decide on when to begin the computer's ascent plan based on two separate sources of information. Recently, more advanced dive computers have integrated air consumption information via long, bothersome air hoses or expensive wireless transmitters.

This paper presents a novel model and control algorithm that prevents the need for a hose or wireless transmitter by indirectly estimating the diver's oxygen consumption using commodity heart rate sensors. We model oxygen consumption as a function of heart rate and prove the safety of our dive control algorithm using the KeYmaera X theorem prover [1].

Our case study demonstrates a method for formally modeling human-in-the-loop control systems for embedded and wearable devices. The two main components in our approach are 1) a model that captures how biological and human behavioral processes interact; and 2) a model of human-computer interaction that leverages a parametric and highly non-deterministic model of the human's decision making in order to provide safety guarantees for all possible human decisions.

\section{BACKGROUND}

Hybrid programs [3] are a programming language for hybrid (discrete + continuous) time dynamical systems. The following example demonstrates how hybrid programs are a nondeterministic imperative programming language extended with ordinary differential equations:

$$
(\underbrace{\left(v:=v_{\text {asc }} \cup v:=0 \cup v:=v_{d e s}\right)}_{\text {ctrl }} ; \underbrace{\left\{d^{\prime}=v \& d \geq 0\right\}}_{\text {plant }})^{*}
$$

The diver makes a discrete choice $(U)$ to ascend $\left(v:=v_{\text {asc }}\right)$, stay at the current depth $(v:=0)$, or descend $\left(v:=v_{d e s}\right)$; then (after the ; operator), the diver follows a single differential equation $\left(d^{\prime}=v\right)$ that continuously updates the diver's depth $(d)$ for any duration that always respects the domain constraint $(d \geq 0)$. This process repeats (by the $*$ operator) any arbitrary number of times.

Reachability properties of hybrid programs are expressible in differential dynamic logic $(\mathrm{d} \mathcal{L})[3]$. The following formula demonstrates how reachability properties are specified in $\mathrm{d} \mathcal{L}$ : 
Table 1: Model Variables and Constraints

\begin{tabular}{lll}
\hline Variable & Meaning & Constraints \\
\hline$h r$ & Heart rate & Physiological limits on $h r: 0<h r_{\text {min }}<h r<h r_{\text {max }}$ \\
$h r_{s s}$ & Steady state heart rate & $h r_{\text {min }}<h r_{s s}<h r_{\text {max }}$ \\
$\tau, b$ & Unitless parameters fit to each diver & $>0$ \\
\hline $\mathrm{d}$ & Depth (distance from surface) & $>0$ \\
stopDepth & Depth of decompression stop to be made during ascent & $>0$ \\
$v$ & Diver's current vertical velocity & Physical limits on $v: v \in\left\{v_{\text {asc }}, 0, v_{\text {desc }}\right\}, v_{\text {asc }}<0 \wedge v_{\text {desc }}>0$ \\
\hline$t$ & Volume of air in the tank & $\geq 0$ \\
$t$ Approx & Controller's approximation of tank & $\geq 0$ \\
\hline c, $\epsilon$ & Time since previous control step, maximum time between control steps & $c \geq 0, \epsilon>0$ \\
stopped & Amount of time the diver has stayed at the stopDepth & $<$ stopTime \\
stopTimer & Amount of time the diver must stay at the stopDepth & $>0$ \\
stON & Boolean flag to check if decompression stop has begun & stON $\in\{0,1\}$ \\
\hline
\end{tabular}

$d \geq 0 \rightarrow\left[(\underbrace{\left(v:=v_{\text {asc }} \cup v:=0 \cup v:=v_{\text {des }}\right)}_{\text {ctrl }} ; \underbrace{\left\{d^{\prime}=v \& d \geq 0\right\}}_{\text {plant }})^{*}\right] d \geq 0$

The above formula states that if the diver starts at a non-negative depth $(d \geq 0)$, then $(\rightarrow)$ after every execution of the hybrid program inside the box [·], the diver will remain at a non-negative depth.

\section{THE MODEL}

Our SCUBA model has three main components: a behavioral model for the diver, a physiological model for the diver, and a mechanism for the diver to occasionally provide ground-truth updates using an analog readout of available breathable air.

Our main result is the following differential dynamic logic [3] theorem, with the meanings of variables given in Table $1:^{1}$

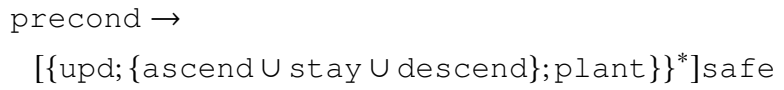

$\left.[\{\text { upd; }\{\text { ascend } \cup \text { stay } \cup \text { descend }\} ; \text { plant }\}\}^{*}\right]$ safe

where:

$$
\begin{aligned}
& \text { upd } \equiv\{\text { NOP } \cup \text { tApprox }:=t\} \\
& \text { ascend } \equiv\left\{\text { ? stopped }=\text { stopTimer } \vee d+\epsilon v_{\text {asc }}>\right.\text { stopDepth; } \\
& h r_{s s}:=* ; ?\left(h r_{\min } \leq h r_{s s} \wedge h r_{s s} \leq h r_{\max }\right) ; \\
& \left.v:=v_{\text {asc }} ; \text { st } O N:=0\right\} \\
& \text { stay } \equiv\left\{? \text { tApprox } \geq \tau h r_{\max } \epsilon+\tau h r_{\max } \frac{-d}{v_{a s c}}\right. \\
& +\tau h r_{\text {max }}(\text { stopTimer - stopped }) \text {; } \\
& h r_{s s}:=* ; ?\left(h r_{\min } \leq h r_{s s} \wedge h r_{s s} \leq h r_{\max }\right) ; \\
& \{? d=\text { stopDepth } \wedge \text { stopTimer }<\text { stopped; } \\
& s t O N:=1 \cup ? s t O N=0\} ; v:=0\} \\
& \text { descend } \equiv\left\{? \text { tApprox } \geq \tau h r_{\max } \epsilon+\tau h r_{\max } \frac{-d-v_{d e s c} \epsilon}{v_{a s c}}\right. \text {; } \\
& \text { ?stopped }=0 ; v:=v_{\text {desc }} ; h r_{s s}:=* \text {; } \\
& \left.?\left(h r_{\text {min }} \leq h r_{s s} \wedge h r_{s s} \leq h r_{\text {max }}\right) ; s t O N=0\right\} \\
& \text { plant } \equiv c:=0 ;\left\{x^{\prime}=b\left(h r_{s s}-x\right), t^{\prime}=-\tau x, d^{\prime}=v, c^{\prime}=1\right. \text {, } \\
& \text { stopped }{ }^{\prime}=\text { stON }
\end{aligned}
$$

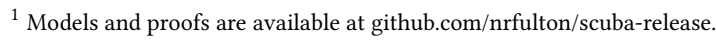

$$
\begin{aligned}
\& d \geq 0 \wedge c \leq \epsilon \wedge \text { stopped } \leq \text { stopTimer }\} \\
\\
\left\{t \text { Approx }:=t \text { Approx }-\tau c h r_{\text {max }}\right\} \\
\text { safe } \equiv(t>0 \wedge(d<\text { stopDepth } \rightarrow \text { stopped } \geq \text { stopTimer }))
\end{aligned}
$$

The diver behavioral model allows the diver to ascend, stay, or descend. Each of these choices has a safety guard and each safety guard assumes a worst-case scenario for the next $\epsilon$ time units assuming the diver's heart rate approaches $\mathrm{hr}_{\max }$. In a concrete implementation of this model, the diver's wrist-mounted computer would display Do Not Descend, Begin Ascent, or Maintain Current Depth advisories. The diver may also choose to make a periodic ground truth update (upd) to the amount of available air in order to cope with worst-cast assumptions.

A physiological model of the dive relating heart rate kinematics to oxygen consumption is central to our approach. Our model is derived from two models proposed by Stirling et al. on heart rate [5] and oxygen update [6] in response to exercise. We make a simplifying assumption that divers stay below the lactate threshold. The diver's oxygen consumption will depend upon both heart rate and several user-specific parameters $\left(b, h r_{s s}\right.$, and $\left.\tau\right)$ fit using data from experiments [4, 7]. Future work will incorporate system identification into this model using [2] and will evaluate a prototype based on our model.

\section{REFERENCES}

[1] Nathan Fulton, Stefan Mitsch, Jan-David Quesel, Marcus Völp, and André Platzer. KeYmaera X: An axiomatic tactical theorem prover for hybrid systems. In $C A D E-25$, volume 9195 of $L N C S$, pages 527-538. Springer, 2015.

[2] Nathan Fulton and André Platzer. Verifiably safe off-model reinforcement learning. In 25th International Conference on Tools and Algorithms for the Construction and Analysis of Systems, Held as Part of the European foint Conferences on Theory and Practice of Software (TACAS 2019), pages 413-430, 2019.

[3] André Platzer. Logical Foundations of Cyber-Physical Systems. Springer, 2018.

[4] Neal Pollock. Aerobic fitness and underwater diving. Fournal of the South Pacific Underwater Medicine Society, 37:118-124, 092007.

[5] James Robert Stirling, Maria Zakynthinaki, Ignacio Refoyo, and Javier Sampedro. A model of heart rate kinetics in response to exercise. Fournal of Nonlinear Mathematical Physics, 15(sup3):426-436, 2008.

[6] J.R. Stirling, M.S. Zakynthinaki, and B. Saltin. A model of oxygen uptake kinetics in response to exercise: Including a means of calculating oxygen demand/deficit/debt. Bulletin of Mathematical Biology, 67(5):989 - 1015, 2005.

[7] David P. Swain, Kimberly S. Abernathy, Shirley J. Smith, Carla S. Lee, and Shelly A. Bunn. Target heart rates for the development of cardiorespiratory fitness. Medicine and science in sports and exercise, 26:112-6, 011994. 